\title{
Large-area flexible Visible-Short wavelength Infrared Photodetection based on III-V compound semiconductor membrane photodetector with high performance
}

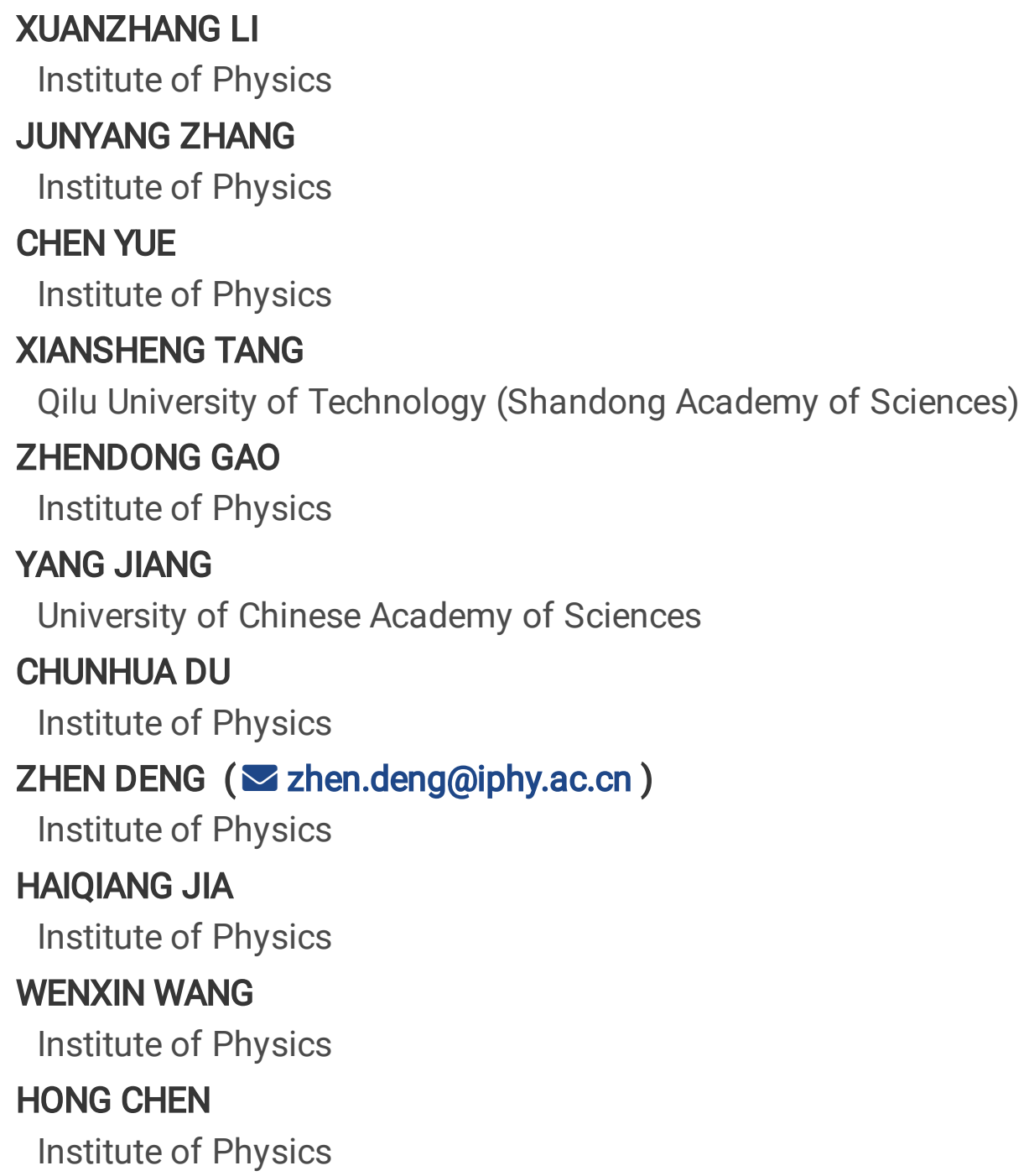

Research Article

Keywords:

Posted Date: March 7th, 2022

DOI: https://doi.org/10.21203/rs.3.rs-1383233/v1 
License: (c) (i) This work is licensed under a Creative Commons Attribution 4.0 International License. Read Full License 


\section{Abstract}

Mechanically flexible optoelectronic devices and systems can enable a much broader range of applications than what their rigid counterparts can do, especially for novel bio-integrated optoelectronic systems, flexible consumer electronics and wearable sensors. Inorganic semiconductor could be a good candidate for the flexible PD when it can keep its high performance under the bending condition. Here, we demonstrate a III-V material-based flexible photodetector operating wavelength from $640 \mathrm{~nm}$ to $1700 \mathrm{~nm}$ with the high detectivity of $5.18 \times 10^{11} \mathrm{~cm} \nabla \mathrm{Hz}^{1 / 2} / \mathrm{W} @ 1550 \mathrm{~nm}$ by using a simply top-to-down fabrication process. The optoelectrical performances are stable as the PDs are exposed to bending cycles with a radius of $15 \mathrm{~mm}$ up to 1000 times. Furthermore, the mechanical failure mode of the PD is also investigated, which suggests that the cracking and delamination failure mode are dominant in bending up and bending down direction, respectively. Such a flexible III-V material-based PD and design with stable and high performance could be a promising strategy for the application of the flexible broad spectrum detection.

\section{Introduction}

Emerging application of mechanically flexible optoelectronic devices have flourished in the fields of flexible imaging/displays $s^{1-5}$, sensors ${ }^{6-8}$, short-reach data communications $s^{9-11}$, solar cell ${ }^{12-14}$ and so on. As an indispensable component of flexible optoelectronic systems, flexible photodetectors (PDs) have been extensively studied and achieved breakthroughs in the kinds of response wavelength ranges based on several material platforms and one of the most important PDs is the visible-infrared broad PD. Organic $\mathrm{PD}$ is the one candidate for next-generation PDs due to their tunable light detection ranges, solution processability, low-cost, lightweight, mechanical flexibility, and seamless heterogeneous integration. A great progress has been achieved for visible or near IR organic PDs, but there are several key bottlenecks, such as large bandgap, weak absorption, and poor charge generation in the NIR region, resulting in poor performance ${ }^{15-17}$. Moreover, the inorganic semiconductor nanowires $(\mathrm{NWs})^{18-20}$, and $2 \mathrm{D}$ materials ${ }^{21}$ have also been applied in the flexible PDs and great progress have been achieved at the visible to shortwavelength IR range because of their unique electronic structure, easy fabrication, broad spectra absorption and flexibility. In the recent years, the devices based on graphene ${ }^{22,23}, \mathrm{PS} /$ graphene $^{24}$, and $\mathrm{HgTe} / \mathrm{Graphene}^{25}$ have been performed to fabricate the flexible broadband PDs. However, the commercial application is limited by their weak light absorption, large noise, high dark current, the stability and reproduction. As a result, those approaches are still some way from being a commercial solution.

Inorganic semiconductor membrane, as an ultrathin layer, is another strong candidate for the flexible PDs because of its excellent flexibility and bendability, while keeping their intrinsic crystal quality and optoelectrical properties. In the application of visible light to short wavelength infrared (SWIR) detection, flexible inorganic PDs have been implemented based on $\mathrm{Si}^{26,27}$ and $\mathrm{Ge}^{28,29}$, but there are still some disadvantages for the broad-spectra high-performance PD. For example, Si membrane PDs are limited in visible wavelength-sensing range and low quantum efficiency due to the property of indirect band gap 
closed to $1 \mathrm{eV}$, while Ge membrane flexible PD shows relatively high dark current attributed to recombination centers located in the damaged Ge. As we know, InGaAs/InP PD has been widely applied and commercialized in the night vision, inspection, agricultural sorting, and so on, due to its broad spectra and high-performance. Thus, in this work, combined with the mature material growth and device fabrication, we have demonstrated the flexible InGaAs membrane PD with the detectivity of $5.18 \times 10^{11}$ $\mathrm{cm} \otimes \mathrm{Hz}^{1 / 2} / \mathrm{W}$ by lifting off epitaxial layers from their native substrates using a sacrificial layer and transferring them onto a flexible host carrier. A sidewall passivation is applied to simplify InGaAs PD fabrication process, and large-area flexible PDs with operating wavelength from $600 \mathrm{~nm}$ to $1700 \mathrm{~nm}$ are obtained. Furthermore, the optoelectrical performance of such flexible PDs keeps stable in bending state and the physical mechanism is also studied in details. As a III-V materials based flexible PD with the conventional high performance of the rigid PDs, one can envision its role for the operation from visible light to short wavelength IR in the novel bio-integrated optoelectronic systems, flexible consumer electronics and wearable sensors.

\section{Methods}

The flexible InGaAs PIN PD fabrication process is schematically illustrated in Fig. 1(a). The process combines constructing metal frame, etching out the release holes, fabricating the passivation layer, peeling off the membranes, depositing the bottom electrode contact and finally transferring the device layers to the flexible organic substrate. Details of the the flexible PD fabrication and characterization are as follows.

\section{Material growth}

Firstly, the III-V PIN PD layers were grown on the 2-inch InP substrate by metal organic chemical vapor deposition. The thickness for the $\mathrm{p}$ - and n-type InP layers were $600 \mathrm{~nm}$ and $500 \mathrm{~nm}$, respectively. There were three lattice matched $\mathrm{In}_{0.53} \mathrm{Ga}_{0.47}$ As layers in the structure, including the top 50-nm-thick p-InGaAs layer as the ohmic contact layer, the $2.2 \mu \mathrm{m}$-thick undoped InGaAs layer as the absorption layer, and the $500 \mathrm{~nm}$-thick $\mathrm{n}$-InGaAs layer between n-InP contact layer and n-InP buffer layer is the sacrificial layer. It is noted that the p-type InGaAs layer and InP layer were achieved by single MOCVD diffusion of zinc after the epitaxy. Then, by using micro/nano fabrication technique, a metal frame layer $(20 \mathrm{~nm} / 200 \mathrm{~nm} \mathrm{Ti} / \mathrm{Au})$ was deposited on the top of the device layers and thus two $150 \mu \mathrm{m}$-wide metal bars formed the metal frame layer. The metal frame layer can be used as the supporting frame to increase the mechanical strength of the to-be released InGaAs-based membrane and the top contact layer for the PD devices.

Device Fabrication

Arrays of $40 \times 40 \mu \mathrm{m}^{2}$ square release holes inside were patterned on the device layers in á100ñ and á010ñ directions, by selectively wet etch down to the surface of the InGaAs sacrificial layer. The release holes were prepared to facilitate the transverse wet etching of InGaAs sacrificial layer beneath the device layer. In the process of selectively wet etching, a $\mathrm{H}_{2} \mathrm{SO}_{4}: \mathrm{H}_{2} \mathrm{O}_{2}: \mathrm{H}_{2} \mathrm{O}(1: 8: 120)$ solution selectively corroded 
InGaAs, but had no effect on InP. On the contrary, the mixed solution of $\mathrm{HCl}_{3} \mathrm{H}_{3} \mathrm{PO}_{4}(1: 3)$ selectively etched InP but has no effect on InGaAs. Therefore, when InGaAs or InP were etched alternately with different etching solution, excessive etching could be properly performed to ensure sufficient corrosion. Based on the selective wet-etching process, the release holes were formed down to the surface of the InGaAs layer (sacrificial layer). The release holes in detail with scan electron microscopy (SEM) were shown in Fig. 2. As the etching rates of á100ñ and á010ñ direction were different ${ }^{30}$, the periodic spacing between the release holes was designed to be $240 \mu \mathrm{m}$ in á010ñ and $290 \mu \mathrm{m}$ in á100ñ, respectively, as shown in Fig. 2. Compared with the same spacing distances in each directions between the release holes ${ }^{31,32}$, the different distances design ensured that the delamination could be completed simultaneously in both directions in the process of the horizontal wet etching separation. Furthermore, As can be seen in Fig. 2 , during the fabrication of release holes, the side length of the square holes expanded from $40 \mu \mathrm{m}$ to 57.5 $\mu \mathrm{m}$ in á100ñ direction and $51.0 \mu \mathrm{m}$ in á010ñ direction, which causes negligible loss to the photosensitive area.

Since the absorption layer was InGaAs layer, the same material as the sacrificial layer, the sidewall of the InGaAs active layer in the release holes need to be protected when the selective etching solution flew to the InGaAs sacrificial layer through the release holes and undergoes etching reaction. Thus, a composite passivation layer was applied on the surface and the sidewall of the devices. According to the reference ${ }^{33,34}, \mathrm{Si}_{3} \mathrm{~N}_{4}$ and $\mathrm{SU} 8$ both play an effective role in improving detector performance, silicon nitride $\left(\mathrm{Si}_{3} \mathrm{~N}_{4}\right)$ can compress dark current promote the detectivity, SU8 can provide better mechanical flexible support. Firstly, a $158 \mathrm{~nm}$-thick $\mathrm{Si}_{3} \mathrm{~N}_{4}$ layer was deposited on the top of the device layers by ICPPECVD and then reactive ion etching was performed to open a $40 \times 40 \mu \mathrm{m}^{2}$ square release hole in the center of each release hole formed by wet etching above, intervals between platforms and the top contact under the $\mathrm{Si}_{3} \mathrm{~N}_{4}$ layer were also etched out. After that, the devices were coated with $1.15 \mu \mathrm{m} \mathrm{SU}$, and then a $26 \times 26 \mu \mathrm{m}^{2}$ square release hole was opened in the center of each release hole by photolithography, the intervals between platforms and the top contact under the $\mathrm{Si}_{3} \mathrm{~N}_{4}$ layer were also exposed again. As shown in the Fig. 2(b) and (c), the shape of release holes coated with SU8 can be recognized as an inverted trapezoid in á010ñ direction and regular trapezoid in á100ñ direction. In general, the passivation layer was confirmed to prevent the InGaAs absorption layer from damage during the wet-etching of sacrificial layer.

Finally, Ti/Au top contacts were covered with photoresist, a temporary protective layer to prevent the device layers from being damaged by metal-assisted chemical etching effect ${ }^{35}$ during the wet-etching of sacrificial layer, and the photoresist layer would be removed after the transfer. After that, the InGaAs sacrificial layer was selectively etched away by immersing the sample in a $\mathrm{H}_{2} \mathrm{SO}_{4}: \mathrm{H}_{2} \mathrm{O}_{2}: \mathrm{H}_{2} \mathrm{O}$ (1:8:120) solution, and the metal framed InGaAs PIN detectors detached from the InP host substrate. Subsequently, the flexible membranes were taken out of the etching solution, and slowly dipped into deionized water to remove residual $\mathrm{H}_{2} \mathrm{SO}_{4}$ and $\mathrm{H}_{2} \mathrm{O}_{2}$. Membranes were flipped and attached to a silicon chip coated with photoresist, then $\mathrm{n}$-contact preparation was accomplished by electron beam evaporation. Finally, InGaAs 
membranes lifted off from the temporary reusable substrate, and a complete flexible InGaAs PD membrane was prepared, which can be installed in any flexible circuit. In our bending experiment, the flexible InGaAs membrane PD were attached to the indium tin oxide (ITO)/PET flexible sheet $(0.05 \mathrm{~mm}$ thick, length $L=35 \mathrm{~mm}$ ) with silver conductive adhesive (about $1 \mu \mathrm{m}$ thick), the whole flexible PD structure was shown in Fig. 1(b). Micrographs of an actual device was shown in Fig. 3(a).

\section{PD Characterization}

The flexible InGaAs PIN PD was measured the current-voltage (I-V) in flat state and bent state at different curvature radius $(R)$. The spectral response of the flexible PDs was carried out by a Fourier transform infrared spectrometer at room temperature in a frontside-illuminated format. All current-voltage (I-V) measurements were performed on micromanipulated probe station using a Keithley 4200 semiconductor characterization system at room temperature. An $1550 \mathrm{~nm}$ wavelength fiber laser with $2 \mu \mathrm{m}$ diameter spot was used as a light source for the I-V curves under illumination characteristics. During the bending test, one end of the PET with a flexible PD was fixed and the other end was pushed forward by $d L$, the bending $R$ is the function of $d L^{36}$, as the model shown in Fig. 3(b). The bending tests of devices were performed in two direction: bending up [Fig. 3(c)] and bending down [Fig. 3(d)] for tensile and compressive strains, respectively.

\section{Results And Discussion}

Figure 4 shows the spectral response ranges of the InGaAs-based membrane PD (Red line) and the InGaAs PIN rigid PD (Black line, as a counterpart) with the same area fabricated from the same epitaxy wafer. It can be clearly seen that the response wavelength of InGaAs-based membrane PD is the same with that of rigid PD in the range of $640 \mathrm{~nm}$ to $1700 \mathrm{~nm}$, which is consistent with other reports ${ }^{37,38}$. The reason is that the InP contact layer of the flexible InGaAs PIN is $600 \mathrm{~nm}$, and the part of the visible light can pass through the contact layer. According to the previous report, thinner InP contact layer between light source and the InGaAs absorption for the visible-IR InGaAs PD can promote the quantum efficiency

in visible light $(400 \mathrm{~nm}-750 \mathrm{~nm})$ and near-IR $(750 \mathrm{~nm}-1000 \mathrm{~nm})$ wavelength region ${ }^{37-39}$ and the PD can detect wavelength as short as $400 \mathrm{~nm}$ with $200 \mathrm{~nm}$-thick InP contact layer, which we will study further and improve our design in the future.

Figure 5(a) and (b) shows the dark current and photocurrent density $(\mathrm{J})$ of the flexible InGaAs membrane PDs in flat condition under various incident optical powers at $1550 \mathrm{~nm}$. It can be clearly seen that the PDs exhibit typical rectifying characteristics, indicating an excellent quality of the PIN junction. The dark current density is approximately $2.50 \mu \mathrm{A} / \mathrm{cm}^{2}$ at $-0.1 \mathrm{~V}$, and there is a gradual increase of the dark current density at the reverse bias region, which could result from the band-to-band tunneling mechanism ${ }^{40}$. A high sensitivity (i.e., the ratio between dark and photocurrent density) of larger than $1.57 \cdot 10^{8}$ is achieved at $1550 \mathrm{~nm}$. In addition, the PD exhibits a linear response with the excitation power increasing from 20 $\mu \mathrm{W}$ to $275 \mu \mathrm{W}$, as shown in Fig. 5(c), indicating that the photogenerated carriers are dominant ${ }^{41,42}$. The responsivity $\left(\mathrm{R}_{\mathrm{i}}\right)$ within the range is $0.53 \mathrm{~A} / \mathrm{W}$ at $-0.1 \mathrm{~V}$, corresponding to an external quantum efficiency 
(EQE) of $42.46 \%$. The Johnson noise limited detectivity D* of the device is calculated according to Ref 43 , 44:

$D^{*}=\frac{q \lambda \cdot E Q E}{h c}\left[2 q J+\frac{4 k_{B} T}{R A}\right]^{-\frac{1}{2}}$

where $q, \lambda, h, c, k_{B}, T, J, R$ and $A$ denote electron charge, laser wavelength, the Planck constant, velocity of light, the Boltzmann constant, operating temperature, dark current density, differential resistance and the effective area of the device, respectively, and it can be extracted from the dark current curve that the $R_{0} A$ of the membrane PD is $2.37 \times 10^{5} \Omega \cdot \mathrm{cm}^{2}$. As a result, the detector exhibits a room temperature $D^{*}$ of $5.18 \times 10^{11} \mathrm{~cm} \varangle \mathrm{Hz}^{1 / 2} / \mathrm{W} @ 1550 \mathrm{~nm}$ at $-0.1 \mathrm{~V}$, whose performance is the same level with the rigid InGaAs SWIR photodetectors ${ }^{45}, 46$. Figure $5(\mathrm{~d})$ shows the photocurrent-time response of the flat PDs under a fixed optical power $(230 \mu \mathrm{W})$, the fall time is about $50.8 \mu$ s and the value is a little longer than the previous report, which will be optimized in future.

In order to study the flexible optoelectrical characteristics, the related characterization for the membrane PDs are performed under the bent condition, while the flat condition is referred as the counterpart. Figure 6 shows the PD's dark current density and photoresponse in their flat and bent up/down state. It should be noted that the dark current density increases gradually under bent down condition [Fig. 6(a)] as $\mathrm{R}$ reduces. Especially when the $R$ is reduced from $15 \mathrm{~mm}$ to $10 \mathrm{~mm}$, the dark current density increases by 5 times. Meanwhile, the phototcurrent density of the device is stable when the radius reduced from $30 \mathrm{~mm}$ to $15 \mathrm{~mm}$, but decreases by $28 \%$ as R is $10 \mathrm{~mm}$. Compared with the bending up condition, the dark current and photocurrent both remain stable under bending down condition, as the radius reduces from $30 \mathrm{~mm}$ to $10 \mathrm{~mm}$.

Furthermore, the failure mode of such PDs have been studied deeply. Optical microscope images as shown in Fig. 7 suggest the failure modes of the flexible InGaAs detectors in two bending directions are different. Under bending up condition, cracks are visible at $\mathrm{R}=10 \mathrm{~mm}$ in Fig. 7(a) and (b), the cracks form perpendicular to the forward direction of $d L$ and propagate from one release hole to another one. The cracks lead to the increase of surface leakage current and the decrease of photocurrent. In addition, once the craking mode occurs, the performance degradation is irreversible. In bending down direction, instead of cracking, we observed that the membrane buckled upward via local delamination from the adhesive at $\mathrm{R}=10 \mathrm{~mm}$, as shown in Fig. 7(c). The delamination reduces the strain in the membrane thereby preventing the cracking failure mode even at smaller bending radius. Therefore, the device can be bent down to $\mathrm{R}=10 \mathrm{~mm}$ while keeping the integrity and stable photo response of the device. As the bending radius decreases further, the delamination continues to expand, as shown in Fig. 7(d). Even if the bending is released, the membranes do not slip back and stay in delamination forever. Instead of the fracture strains of membranes suffering, the interfacial shear stresses are responsible for the failure behavior and the delamination failure is sensitive to the adhesion strength of film to the substrate ${ }^{36}$. Hence, increasing the adhesion strength of InGaAs membrane PD to PET helps to overcome delamination. 
According to the study about the failure mode of bending silicon ribbons on plastic substrates, the thickness influences the bending mechanics and determines the dominant failure mode, as the thickness increases, the dominant failure mode is as follows: cracking, slipping and delamination ${ }^{36,47,48}$. However, there are two failure modes for the flexible InGaAs PD with a fixed thickness in two bending direction. Unlike the silicon ribbon, the flexible InGaAs PD is a composite multilayer asymstructre, the effect of bending strain on each layer of material is different. As shown in Fig. 1(b), SU8 layer, $\mathrm{Si}_{3} \mathrm{~N}_{4}$ layer and the metal contact coated on the InGaAs PIN membrane structure, asymmetrically from top to bottom. The failure mode is supposed to occur from one layer and transmit to the whole structure. Top and bottom thin metal contact has ductility, and its contribution to the failure membranes can be neglected. The comparison of the Young's moudlus for SU8, $\mathrm{Si}_{3} \mathrm{~N}_{4}$ and InGaAs PIN membrane is: SU8 (3-5 GPa) < InGaAs PIN membrane (60-80 GPa) $<\mathrm{Si}_{3} \mathrm{~N}_{4}(220-230 \mathrm{GPa})$. The failure mode is more likely to occur in InGaAs PIN membrane and $\mathrm{Si}_{3} \mathrm{~N}_{4}$. Considering the thickness of the three materials, it can be assumed that, cracking failure dominated in bending up caused by thin $\mathrm{Si}_{3} \mathrm{~N}_{4}$, and the delamination failure dominated in bending down caused by thick InGaAs PIN expitaxy layer. The quantitative analysis of this hypothesis can be further studied, and provide guidance for the bending performance optimization.

In general, the ratio between dark and photocurrent density of the PDs remains stable, larger than $7 \cdot 10^{8}$ under bending up condition with $\mathrm{R}$ decreasing from $30 \mathrm{~mm}$ to $15 \mathrm{~mm}$ and bending down condition with $\mathrm{R}$ decreasing from $30 \mathrm{~mm}$ to $10 \mathrm{~mm}$, respectively, indicating that the simplify fabrication of flexible InGaAs PIN membrane PD is feasible.

Fatigue test of the flexible InGaAs-based PD is further performed with a self-assembled slide stable system. The PD is bent on the automated slide rail for 1,10,100, and 1000-times bending down cycles, respectively. As shown in Fig. 8 , the ratio of photo to dark current density remain more than $4 \cdot 10^{8}$, while the responsivity remains above $0.49 \mathrm{~A} / \mathrm{W}$ and thus no failure occur after the bending cycle. These results suggest that such PDs have an excellent durability of the optoelectrical performance after multiple mechanical deformations. Therefore, it could be believed that such gratifying robustness of the flexible InGaAs-based PD with the simplified fabrication process is promising to be applied in the flexible optoelectronic field.

\section{Conclusion}

In this paper, we have demonstrated the flexible InGaAs-based membrane PD with the detectivity of $5.18 \times 10^{11} \mathrm{~cm} \otimes \mathrm{Hz}^{1 / 2} / \mathrm{W}$ by a top-to-down fabrication technique using a sacrificial layer and transferring them onto a flexible host carrier. A side wall passivation with SU8 and $\mathrm{Si}_{3} \mathrm{~N}_{4}$ is applied to simplify InGaAs PD fabrication process. And a large-area flexible PD with operating wavelength from $640 \mathrm{~nm}$ to $1700 \mathrm{~nm}$ is obtained and the response time is about $50.8 \mu \mathrm{s}$. Furthermore, the results reveal that the over-all optoelectrical performance of such flexible PDs keeps stable in bending state. In addition, fatigue test suggests that cracking and delamination modes dominates in bending up and down conditions, respectively. We believe that the demonstrated flexible III-V material-based PD with the high performance 
of the rigid PD is step forward in merging mature compound semiconductor technology and flexible optoelectronic device with simple design and high performance for broad spectrum detection.

\section{Declarations}

\section{Acknowledgements}

This work is supported by the National Natural Science Foundation of China (Grant Nos. 62004218, and 61991441), Youth Innovation Promotion Association, Chinese Academy of Sciences (Grant No. 2021005), and the Strategic Priority Research Program of Chinese Academy of Sciences (Grant No. XDB01000000). This work is also supported by the Center for Clean Energy, Institute of Physics, Chinese Academy of Sciences.

\section{Author Contributions}

X.L. and Z.D. conceived the idea. X.L. conducted the device fabrication. J.Z., C.Y., X.T. and Z.G. equally to this work for assisting the sample preparation and characterization. H.C. supervised the entire research project. The manuscript was written by X.L. and Z.D. All authors have reviewed the manuscript and given their approval to the final version of this manuscript.

\section{Additional Information}

Competing financial interests: The authors declare no competing financial interests.

\section{Data availability}

All data generated or analysed during this study are included in this published article (and its Supplementary Information files).

\section{References}

1. Forrest, S.R. The path to ubiquitous and low-cost organic electronic appliances on plastic. Nature 428, 911-918 (2004).

2. Chien, L.-C., Okuda, Y., Lee, S.-D., Fujieda, I. \& Wu, M.H. in Advances in Display Technologies II (2012).

3. Seo, J.-H. et al. A Simplified Method of Making Flexible Blue LEDs on a Plastic Substrate. IEEE Photonics Journal 7, 1-7 (2015).

4. Park, J. et al. Research on flexible display at Ulsan National Institute of Science and Technology. npj Flexible Electronics 1 (2017).

5. Choi, M.K., Yang, J., Hyeon, T. \& Kim, D.-H. Flexible quantum dot light-emitting diodes for nextgeneration displays. npj Flexible Electronics 2 (2018).

6. Park, J. et al. Giant tunneling piezoresistance of composite elastomers with interlocked microdome arrays for ultrasensitive and multimodal electronic skins. ACS Nano 8, 4689-4697 (2014). 
7. Salvatore, G.A. et al. Biodegradable and Highly Deformable Temperature Sensors for the Internet of Things. Advanced Functional Materials 27 (2017).

8. Ha, M., Lim, S. \& Ko, H. Wearable and flexible sensors for user-interactive health-monitoring devices. J Mater Chem B 6, 4043-4064 (2018).

9. Bosman, E. et al. Highly Reliable Flexible Active Optical Links. IEEE Photonics Technology Letters 22, 287-289 (2010).

10. Dangel, R. et al. Development of Versatile Polymer Waveguide Flex Technology for Use in Optical Interconnects. Journal of Lightwave Technology 31, 3915-3926 (2013).

11. Li, L. et al. A Fully-Integrated Flexible Photonic Platform for Chip-to-Chip Optical Interconnects. Journal of Lightwave Technology 31, 4080-4086 (2013).

12. Fu, X., Xu, L., Li, J., Sun, X. \& Peng, H. Flexible solar cells based on carbon nanomaterials. Carbon 139, 1063-1073 (2018).

13. Shin, D.H., Jang, C.W., Lee, H.S., Seo, S.W. \& Choi, S.H. Semitransparent Flexible Organic Solar Cells Employing Doped-Graphene Layers as Anode and Cathode Electrodes. ACS Appl Mater Interfaces 10, 3596-3601 (2018).

14. Fan, Z. et al. Three-dimensional nanopillar-array photovoltaics on low-cost and flexible substrates. Nat Mater 8, 648-653 (2009).

15. Tordera, D. et al. in Organic Flexible Electronics 575-597 (2021).

16. Xu, H. et al. Flexible Organic/Inorganic Hybrid Near-Infrared Photoplethysmogram Sensor for Cardiovascular Monitoring. Adv Mater 29 (2017).

17. Khan, Y. et al. A flexible organic reflectance oximeter array. Proc Natl Acad Sci U S A 115, E11015E11024 (2018).

18. Zhe et al. Fabrication of high-quality ZnTe nanowires toward high-performance rigid/flexible visiblelight photodetectors. 21 (2013).

19. Chen, G. et al. High performance rigid and flexible visible-light photodetectors based on aligned X(In, Ga)P nanowire arrays. J. Mater. Chem. C 2, 1270-1277 (2014).

20. Dahiya, A.S. et al. High-performance printed electronics based on inorganic semiconducting nano to chip scale structures. Nano Converg 7, 33 (2020).

21. De Fazio, D. et al. High Responsivity, Large-Area Graphene/MoS2 Flexible Photodetectors. ACS Nano 10, 8252-8262 (2016).

22. Kang, P., Wang, M.C., Knapp, P.M. \& Nam, S. Crumpled Graphene Photodetector with Enhanced, Strain-Tunable, and Wavelength-Selective Photoresponsivity. Adv Mater 28, 4639-4645 (2016).

23. Goossens, S. et al. Broadband image sensor array based on graphene-CMOS integration. Nature Photonics 11, 366-371 (2017).

24. Yousefi, R. et al. Effect of annealing temperature and graphene concentrations on photovoltaic and NIR-detector applications of PbS/rGO nanocomposites. Ceramics International 42, 15209-15216 (2016). 
25. Noumbe, U.N. et al. Reconfigurable 2D/OD p-n Graphene/HgTe Nanocrystal Heterostructure for Infrared Detection. ACS Nano 14, 4567-4576 (2020).

26. Seo, J.H. et al. Flexible Phototransistors Based on Single-Crystalline Silicon Nanomembranes. Advanced Optical Materials 4, 120-125 (2015).

27. Moriceau, H., Fournel, F. \& Rieutord, F. in Silicon-On-Insulator (SOI) Technology 3-51 (2014).

28. Ho, W.S. et al. Flexible Ge-on-polyimide detectors. Applied Physics Letters 94 (2009).

29. Yoshimine, R., Toko, K., Saitoh, N., Yoshizawa, N. \& Suemasu, T. Silver-induced layer exchange for polycrystalline germanium on a flexible plastic substrate. Journal of Applied Physics 122 (2017).

30. Pasquariello, D. et al. Selective undercut etching of InGaAs and InGaAsP quantum wells for improved performance of long-wavelength optoelectronic devices. Journal of Lightwave Technology 24, $1470-$ 1477 (2006).

31. Kiefer, A.M. et al. Si/Ge Junctions Formed by Nanomembrane Bonding. ACS Nano 5, 1179-1189 (2011).

32. Weiquan Yang et al. Large-area InP-based crystalline nanomembrane flexible photodetectors. Applied Physics Letters (2010).

33. Li, J. et al. in AOPC 2017: Optical Sensing and Imaging Technology and Applications (2017).

34. Kim, H.S. et al. Performance improvement of InAs/GaSb strained layer superlattice detectors by reducing surface leakage currents with SU-8 passivation. 96, 033502 (2010).

35. Kong, L. et al. Damage-Free Smooth-Sidewall InGaAs Nanopillar Array by Metal-Assisted Chemical Etching. ACS Nano 11, 10193-10205 (2017).

36. Park, S.-I. et al. Theoretical and Experimental Studies of Bending of Inorganic Electronic Materials on Plastic Substrates. Advanced Functional Materials 18, 2673-2684 (2008).

37. Andresen, B.F. et al. in Infrared Technology and Applications XXX (2004).

38. Andresen, B.F. et al. in Infrared Technology and Applications XXXIV (2008).

39. Cohen, M.J., Lange, M.J., Ettenberg, M.H., Dixon, P. \& Olsen, G.H. in LEOS '99. IEEE Lasers and ElectroOptics Society 1999 12th Annual Meeting (1999).

40. Huang, Y.H., Chang, G.E., Li, H. \& Cheng, H.H. Sn-based waveguide p-i-n photodetector with strained GeSn/Ge multiple-quantum-well active layer. Opt Lett 42, 1652-1655 (2017).

41. Li, L. et al. High-performance flexible waveguide-integrated photodetectors. Optica 5 (2018).

42. An, S. et al. High-Sensitivity and Mechanically Compliant Flexible Ge Photodetectors with a Vertical p-i-n Configuration. ACS Applied Electronic Materials 3, 1780-1786 (2021).

43. Haddadi, A. et al. High-performance short-wavelength infrared photodetectors based on type-Il InAs/InAs1-xSbx/AIAs1 - xSbx superlattices. Applied Physics Letters 107 (2015).

44. Liu, J. et al. InAs/InGaAs/InAlAs interband quantum well infrared photodetector (IQWIP) with cut-off response wavelength at $1.93 \mu \mathrm{m}$. Applied Physics Express 12, 032005 (2019).

45. Shao, X. et al. Developments of High Performance Short-wave Infrared InGaAs Focal Plane Detectors. (2016). 
46. Xie, Z., Deng, Z., Zou, X. \& Chen, B. InP-Based Near Infrared/Extended-Short Wave Infrared Dual-Band Photodetector. IEEE Photonics Technology Letters 32, 1003-1006 (2020).

47. Huang, Y., Feng, X. \& Qu, B. Slippage toughness measurement of soft interface between stiff thin films and elastomeric substrate. Rev Sci Instrum 82, 104704 (2011).

48. Dai, L., Yin, H., Hang, C., Xue, F. \& Fang, D. Transition among failure modes of the bending system with a stiff film on a soft substrate. Applied Physics Letters 106, 021905.021901-021905.021904 (2015).

\section{Figures}

\section{Figure 1}

Device fabrication: (a) A flow chart for fabrication process: (i) Material growth of InGaAs PIN PD, (ii) Deposition of Up-contact metal frame. (iii) Preparation of release holes array, (iv) Preparation of passivation layer, (v) Preparation of electrode protective layer, (vi) Peeling off the InGaAs PIN membrane PD, (vii) Deposition of bottom contact and transfer of devices. (b) Cross-sectional structure of a fabricated InGaAs PIN PD based on ITO/PET flexible substrate.

\section{Figure 2}

SEM image of etch holes in fabricated InGaAs PD based on the InP substrate: (a) The surface of the etch holes array. $(b, c)$ The cross section of side wall covered with composite passivation layer in [100] and [010] direction. (d) A magnified view of a side wall of the outline area on the left shows side wall covered with $\mathrm{Si}_{3} \mathrm{~N}_{4}(158 \mathrm{~nm})$ and SU8 $(1.15 \mu \mathrm{m})$.

\section{Figure 3}

(a) Zoom-in views of a fabricated large area $\left(3^{\prime} 3 \mathrm{~mm}^{2}\right)$ InGaAs PD on flexible PET substrate. (b) Schematic diagram for the measurement principle and the corresponding model. (c, d) A schematic diagram of fabricated flexible InGaAs PD under bending up and bending down test, respectively. The bottom left insets provide schematic illustrations of the bending geometries. 


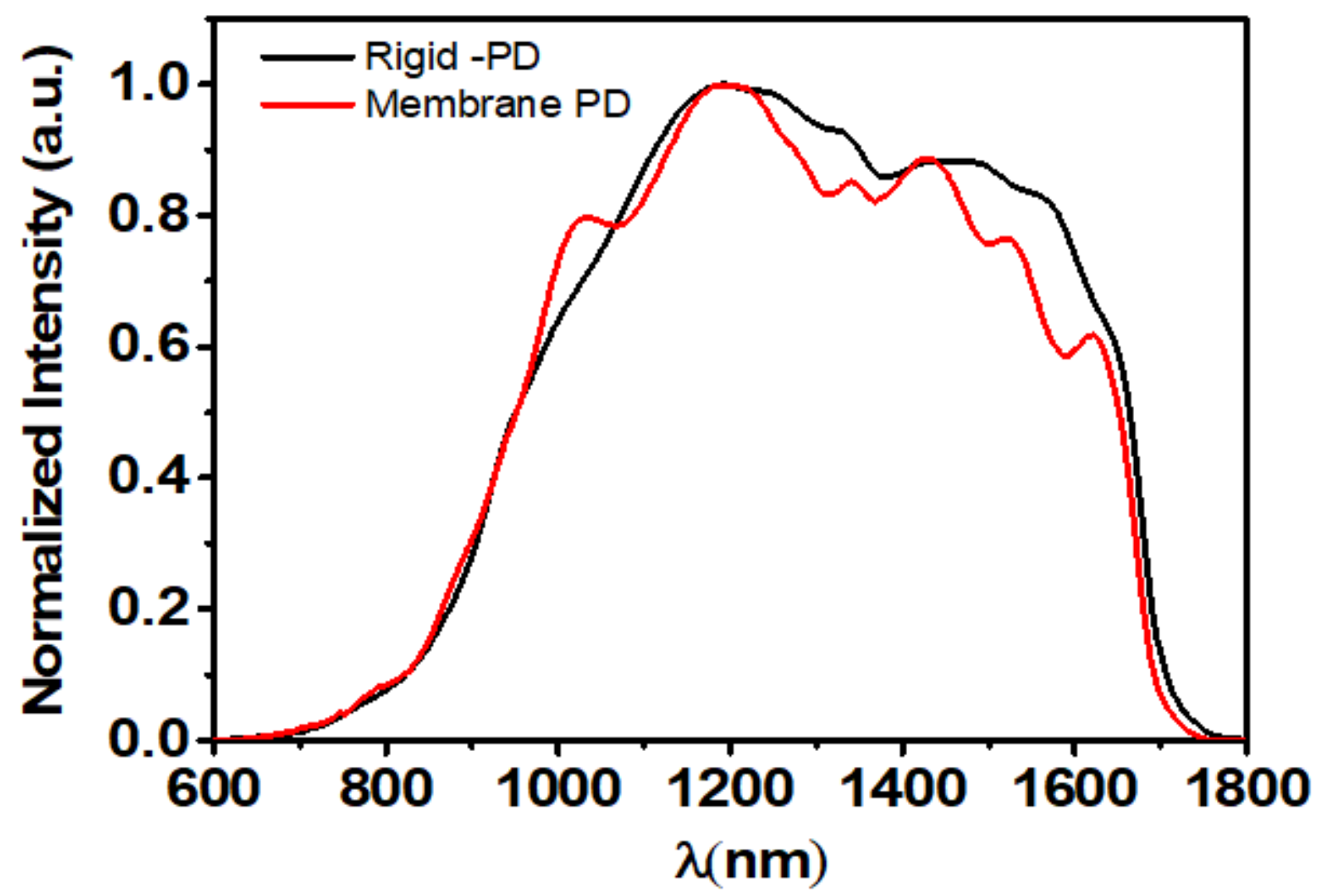

Figure 4

The response Spectrum of InGaAs Flexible membrane PD and InGaAs rigid PD at 300K

Figure 5

J-V responses of the InGaAs flexible detector under flat condition: (a) At darkness and (b) Different input optical power levels. (c) Photocurrent (PC) as a function of the optical power, the solid line represents a linear fitting of the experimental data. (d) Dynamic photocurrent behavior.

Figure 6

Device characterization under bent conditions: J-V responses of the detector under bending up and down condition. At darkness: $(a, c)$ and Illumination: $(b, d)$ at $1550 \mathrm{~nm}(P=230 \mu \mathrm{W})$. 


\section{Figure 7}

Microscope image of bending flexible InGaAs detector: (a) Bending up of $R=10 \mathrm{~mm}$. (b) A magnified view of the outline area on the left shows the cracking failure surface. (c) Bending down of $R=10 \mathrm{~mm}$ shows the appearance of the delamination failure. (d) Bending up of $R<10 \mathrm{~mm}$ shows the expansion of the delamination failure.

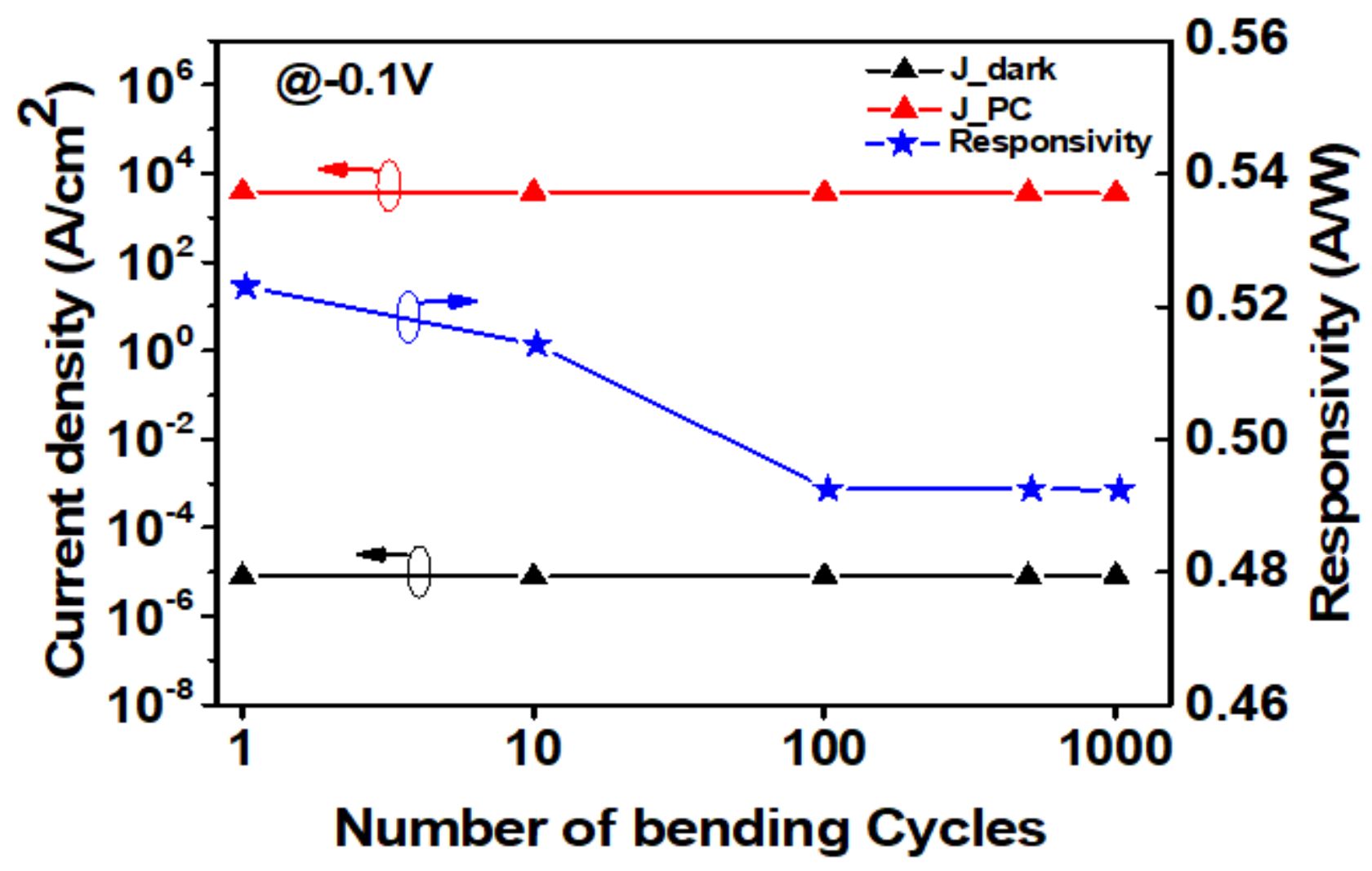

Figure 8

Devices charaterization after multiple bending down cycle of $1,10,100,1000$ times (a radius of $R=15$ $\mathrm{mm}$ ): Left: Dark and photocurrent (PC) density of the devices. Right: Responsivity of the device at 1550 $\mathrm{nm}(\mathrm{P}=230 \mu \mathrm{W})$.

\section{Supplementary Files}

This is a list of supplementary files associated with this preprint. Click to download.

- SCSupplement.docx 